\title{
Loss of mitochondrial transmembrane potential and caspase-9 activation during apoptosis induced by the novel styryl-lactone goniothalamin in HL-60 leukemia cells
}

\begin{abstract}
Styryl-lactones such as goniothalamin represent a new class of compounds with potential anti-cancer properties. In this study, we investigated the mechanisms of goniothalamin (GTN), a plant styryl-lactone induced apoptosis in human promyelocytic leukemia HL-60 cells. This plant extract resulted in apoptosis in HL-60 cells as assessed by the externalisation of phosphatidylserine. Using the mitochondrial membrane dye (DIOC6) in conjunction with flow cytometry, we found that GTN treated HL-60 cells demonstrated a loss of mitochondrial transmembrane potential ( $9 q_{\mathrm{g}} \mathrm{m}$ ). Further immunoblotting on these cells showed activation of initiator caspase-9 and the executioner caspases-3 and -7. Pretreatment with the pharmacological caspase inhibitor, benzyloxycarbonyl-Val-Ala-Asp fluoromethyl ketone (ZVAD.FMK) abrogated apoptosis as assessed by all of the apoptotic features in this study. In summary, our results demonstrate that goniothalamin-induced apoptosis occurs via the mitochondrial pathway in a caspase dependent manner.
\end{abstract}

Keyword: Apoptosis; Caspase activation; Goniothalamin; Mitochondrial transmembrane potential; Styryl-lactone 